\title{
Geschichtsdarstellung in der Gegenwartsliteratur: Florian Illies' Pop-Chronik der Welt von Gestern
}

[Representation of History in Contemporary Literature: Florian Illies's PopChronicle of the Belle Epoque]

\begin{abstract}
Florian Illies' book 1913. Der Sommer des Jahrhunderts (1913. The Year before the Storm) has been a major success with critics and readers. Based on documented facts, the author tells a kind of cultural history of the last year before the Great War, formed by independent episodes from the lives of innumerable persons, mainly artists, writers and other celebrities. Using his imagination and techniques of fictional writing the text shows remarkable proximity to fiction although it does not invent characters or events. The article compares the book to other recent literary representations of history and analyses its narrative structure. It will be shown that the author chose a casual ironical style and an intimate perspective on the protagonists in order to entertain the reader with elements still relevant in our contemporary popular culture. It seems to be intended that the reader develops a kind of counterfactual fantasy that links his present directly to the Golden Age of art, omitting the catastrophes of 20th century.
\end{abstract}

Key-words: Florian Ilies; Historical novel; Pop Literature; Fiction; Cultural History

Resumo: O livro 1913. Der Sommer des Jahrhunderts (1913. O verão do século) de Florian Illies foi um dos maiores sucessos recentes junto à crítica e os leitores. Baseado em fatos documentados o autor conta uma espécie de história cultural do último ano antes da Grande Guerra, formada por episódios independentes das vidas de inúmeras pessoas, sobretudo artistas, escritores e outros proeminentes. Usando sua imaginação e técnicas da escrita ficcional, o texto apresenta uma proximidade notável da ficção apesar de não inventar personagens $\mathrm{e}$ acontecimentos. O presente artigo compara o livro com outras representações literárias da história recentes e analisa sua estrutura narrativa. Será mostrado que o autor elegeu um estilo descontraído e irônico e uma perspectiva íntima sobre os protagonistas para entreter o leitor com elementos que ainda são relevantes na nossa cultura popular contemporânea. A intenção do autor, ao que parece, é fazer com que o leitor desenvolva uma espécie de fantasia contrafactual que o relacione diretamente com esse Século de Ouro da arte, pulando as catástrofes do século $\mathrm{XX}$.

Palavras-chave: Florian Illies; Romance histórico; Literatura Pop; Ficção; História cultural

\footnotetext{
1 Professor für Deutsche Literatur an der Universität São Paulo. Email: helmut_galle@hotmail.com

Pandaemonium, São Paulo, v. 17, n. 23, Jun./2014, p. 26-49
} 
Galle, H. -Geschichtsdarstellung in der Gegenwartsliteratur

\section{Aktuelle Tendenzen: historischer Roman, historiographische Metafiktion und Dokumentencollage}

Der historische Roman hat gegenwärtig Konjunktur. Die ,triviale“ Variante füllt die entsprechenden Regale der Buchläden mit hunderten von Titeln und die anspruchsvolle wird prämiert wie der Roman Landgericht von Ursula Krechel mit dem Buchpreis 2012. Gleichzeitig ist das Genre in Bewegung. Zwar gibt es noch immer viele Autoren wie Ken Follett, die im Großen und Ganzen dem von Walter Scott gestifteten Modell nacheifern und Geschichten von fiktiven Personen vor dem Hintergrund (mehr oder weniger) sorgfältig recherchierter historischer Ambientes erzählen.

Die ambitionierteren Autoren jedoch hatten der im Kontext der Moderne lange missachteten Gattung in den vergangenen Jahrzehnten eine neue Dignität gegeben, indem sie einerseits die Reflexion auf die sprachliche Bedingtheit alles historischen Erzählens als auch andererseits die Lust an den Geschichten wieder zur Geltung brachten. „Historiographische Metafiktion“ nannte Linda HUTCHEON (1991: 105ff.) diese Richtung der postmodernen Literatur, zu der sie Autoren wie Umberto Eco, E. L. Doctorow und John Fowles zählte und die geradezu zur Leitgattung der Postmoderne avancierte. Charakteristisch für das Genre war, dass den Autoren und ihren Erzählern das Vertrauen in Wahrheiten abhanden gekommen war, und zwar nicht nur das Vertrauen in die Wahrheit der Historiographie, sondern auch das in die Wahrheit der Fiktion, das Franz Werfel 1924 noch als Motto über seinen Verdi. Roman der Oper gesetzt hatte. Die historischen Romane der Zwanziger und Dreißiger Jahre setzen noch ganz auf die autonome Schöpfung der Vergangenheit durch den Autor, zu deren wichtigsten neben Werfel, den Brüdern Mann, und Alfred Döblin vor allem Stefan Zweig gehörte. Zweig hatte dann, ins Exil getrieben und von Resignation gezeichnet, seine eigene Lebenszeit als Die Welt von Gestern (posthum: 1942) dargestellt: die Welt der Bürger und Literaten vor dem Ersten Weltkrieg, eine Welt der Verständigung zwischen den europäischen Völkern und der Kunst, die er in der Katastrophe der beiden Weltkriege unwiderruflich untergehen sah. Das Jahr 1913, das, ohne dass die Menschen es ahnen konnten, den letzten Höhepunkt dieser langen Phase des Friedens und kultureller Blüte darstellte, wird das Thema des in den folgenden Kapiteln untersuchten Buches von Florian Illies. 
Galle, H. -Geschichtsdarstellung in der Gegenwartsliteratur

Der in den vergangenen Dekaden dominierende postmoderne Roman setzt nicht auf die eine, sondern auf eine Vielzahl von Wahrheiten, die sich gegenseitig entkräften, und dies nicht nur auf thematischer, sondern auch formaler Ebene. Die Ironie der metafiktionalen Einwürfe des Erzählers dient hier vor allem dazu, den Realitätsgehalt der Erzählung in Zweifel zu ziehen und dies bezieht sich gleichermaßen für die Wahrheit des Erzählten in der Fiktion wie für die Wahrheit der extratextuellen Erzählungen von der Geschichte.

Im deutschsprachigen Kontext wurden in diesem Zusammenhang meist die Namen von Patrick Süskind, Sten Nadolny und Christoph Ransmayr genannt (vgl. GRIMM 2008; SCHILLING 2012). Auch Daniel Kehlmanns großer internationaler Erfolg Die Vermessung der Welt könnte als Beispiel für den postmodernen Geschichtsroman verstanden werden, insofern das Buch Ironie als zentrales Stilmittel kultiviert, die historischen Akteure A. v. Humboldt und C. F. Gauß als Protagonisten auftreten und in ihren Äußerungen über Literatur metafiktionale Kommentare aufscheinen. Allerdings richtet sich die Ironie nicht auf die eigenen sprachlich-literarischen Grundlagen des Romans, sondern auf seine historischen Objekte, zwei „Monumente“ der Wissenschaftsgeschichte und deren vermeintlich „,vermessenden“, naturwissenschaftlich rationalen Blick auf die Welt: Gauß und Humboldt erscheinen eher als Karikaturen, deren persönliche Deformationen die wissenschaftlichen Leistungen überwiegen und den Leser dazu einladen, sich mit dem Erzähler über diese „Geistesheroen“ zu amüsieren.

Wie Friedhelm Marx bemerkte, entlarvt der Roman „die Fiktion im scheinbar Authentischen und behauptet dagegen das Authentische der Fiktion“ (MARX 2008: 178). Dieses Vertrauen auf die Fiktion kann allerdings schon als Schritt über die postmoderne Metafiktion hinaus gesehen werden, denn Kehlmann spielt zwar mit der historischen Wahrheit, insofern seine Protagonisten deutlich von ihren historischen Vorbildern abweichen (vgl. OORT 2008; ETTE 2012; Holl 2012; KNOBLOCH 2012), aber seine Erzählung stellt die von ihr behauptete Wahrheit nicht selbst in Frage. Insofern ist Erik Schilling zumindest teilweise recht zu geben, wenn er urteilt: „Keine postmoderne Pluralität, keine ironisch gebrochene Aussage, keine Zweifel am Status des eigenen Subjekts - stattdessen die Grundlage eines neuen Paradigmas im Felde des historischen Romans.“ (ScHILling 2011: 255). Ob es sich in der Tat um ein neues Paradigma handelt, muss sich allerdings erst noch erweisen. 
Galle, H. -Geschichtsdarstellung in der Gegenwartsliteratur

Seit der Wende von 1989 hatte die postmoderne Variante des Geschichtsromans ohnehin schon Konkurrenz bekommen von Autoren, denen es gar nicht so sehr um die Selbstreferenz als vielmehr um die Referenz auf historische Quellen und Dokumente ging. Seit Anfang der 90er Jahre erschien das monumentale Echolot-Projekt von Walter Kempowski, das in 10 Bänden die Stimmen von hunderten von Tagebuch- und Briefschreibern zu einem ,kollektiven Tagebuch“ zusammenführt. Zwar handelt es sich hier nicht mehr um einen Roman im herkömmlichen Sinn, sondern zunächst um ein „Archiv“ des zeitgenössischen Diskurses im Sinne BAßLERS (2002), aber in seiner spezifischen Form entfaltet es eine sehr ähnliche Wirkung auf den Leser wie eine fiktionale Erzählung. Die persönlichen Mitteilungen der militärischen und Zivilpersonen bewirken eine affektive Teilnahme des Lesers an den Schicksalen der realen Protagonisten, die sich nicht nur vor dem Hintergrund des historischen Geschehens bewegen, sondern mitten darin. Gattungsmäßig ist Kempowskis Werk insofern hybrid, als es zwar authentische historische Quellen bietet, diese aber nicht historiographisch zu einer Erzählung verarbeitet, sondern in ihrer Synchronizität, Vielstimmigkeit und Widersprüchlichkeit nebeneinanderstellt, wie Baßler (2002) herausgestellt hat. Dass Kempowski dabei nicht nur als Herausgeber und Kompilator, sondern in einem klassischen Sinne als Autor fungiert - wenn auch verschleiert -, das haben inzwischen Vergleiche der Buchfassung mit dem Archivmaterial belegen können. Wie PEREIRA (2011) zeigen konnte, bewirkt die Auswahl und Kürzung der Quellen, dass der Leser sich die einzelnen Stimmen historischen Typen zuordnet, die von Kempowski in einer bestimmten Konstellation angeordnet werden, die letztlich als „große Erzählung“ fungiert, auch wenn dies nicht ohne weiteres erkennbar wird. Es ist also nicht abwegig, hier von einem historischen Roman zu sprechen, auch wenn keine eigentlich fiktionalen Gehalte - im Sinne von Erfundenem - vorliegen. Dennoch handelt es sich fraglos um eine neuartige Form der Darstellung von Geschichte, die sich von den herkömmlichen historiographischen Narrativen und von denen des historischen Romans verabschiedet.

Hatte es zunächst so ausgesehen, als müsse das Echolot ein Solitär bleiben, so ist seither doch eine Reihe von Büchern erschienen, die ähnlich strukturiert sind, sei es dass die unterschiedlichsten Stimmen aus einer bestimmten Phase des 2. Weltkrieges als kollektives Tagebuch zusammengeschnitten werden wie Nicolas BAKERS Menschenrauch (Human Smoke, 2008), sei es dass eine große Zahl von (vor allem 
Galle, H. -Geschichtsdarstellung in der Gegenwartsliteratur

historischen) Biographien zusammengeführt und nacherzählt werden wie in William T. Vollmanns Europe Central (2005), oder dass die Chronik eines Gettos mit einer Mischung aus geschichtsbasierter Erzählung und Dokumentenmontage dargestellt wird wie in Steve Sem-SandBergs Die Elenden von Lodz (2009). All diesen Büchern ist noch gemeinsam, dass es in ihnen um die Katastrophe geht: den Weltkrieg, den Genozid, das Leben und Sterben unter den Bedingungen des totalitären Staates.

Dies ist anders in 1926. Ein Jahr am Rand der Zeit, mit dem Hans Ulrich GUMBRECHT schon 1997 (amerikanische Ausgabe) versucht hatte, eine „Momentaufnahme“ der europäischen Kultur nach der Vernichtungsorgie des 1. Weltkriegs und vor dem Machtantritt der Nazis herzustellen, die allerdings eher wissenschaftlich als literarisch ausgerichtet und nach Sachthemen in der Art einer Internet-Enzyklopädie verlinkt ist, anstatt chronologischer Folge ein Register der „Dispositive“ und „Codes“ jener Zeit. Aber auch Gumbrecht will den Leser eintauchen lassen in das Archiv der historischen Realität, damit er seine Gegenwartsperspektive zeitweise hintansetzt und erkennt, wie „offen“ die Horizonte im Jahr 1926 - auf der Höhe des Booms der „Goldenen“ 20er Jahre und der Neuen Sachlichkeit und vor der großen Krise von 1929 - waren und wie wenig sich damals voraussehen ließ, was wenige Jahre später geschah und was aus heutiger Sicht wie eine „notwendige“ und unabänderliche Entwicklung erscheint, einfach weil es geschehen ist. Ähnlich wie Kempowski lässt Gumbrecht die Dokumente sprechen und zitiert oder referiert zum größten Teil öffentliche Quellen, die dem Leser wie in den andern angesprochenen Büchern in umfangreichen Quellenverzeichnissen nachprüfbar gemacht sind. Gumbrecht will allerdings nicht unterhalten, sondern es geht ihm primär um historische Erkenntnis, eine Erkenntnis, die aus Kontextualisierung und aus der Gleichzeitigkeit von Wichtigem und Unwichtigem entsteht und nicht, wie die gängigen historiographischen Narrative aus der Chronologie von Ursache und Wirkung, der Hierarchisierung von Bedeutendem und Unbedeutendem im retrospektiven Urteil. 


\section{Florian Illies' 1913. Der Sommer des Jahrhunderts: Hybrid zwischen Sachbuch und Fiktion}

Das Ende 2012 publizierte Buch 1913. Der Sommer des Jahrhunderts von Florian Illies ist eines der meistverkauften deutschen Bücher der letzten 20 Jahre (1 Million Exemplare bis Januar 2014), wurde bereits in mehr als 6 Sprachen übersetzt und stand seit seinem Erscheinen monatelang auf der Bestsellerliste des Spiegel. Es scheint mit den zuvor beschriebenen, zwischen Literatur und Geschichtsschreibung angesiedelten hybriden Beispielen der „sogenannten synoptischen Geschichtsschreibung“ (TROTHA 2012) vieles gemein zu haben: Es ist nicht als kohärente Erzählung konzipiert, sondern präsentiert unzusammenhängende Realitätspartikel in Form von nach Monaten strukturierten Annalen; es greift auf ein Archiv von Dokumenten zurück; es bewegt sich in einer Grauzone zwischen Fiktion und Historiographie.

1913 trägt keine Gattungsbezeichnung, wird aber im S. Fischer Verlag und in den meisten Besprechungen als „Sachbuch“ geführt. Die Klassifikation scheint durch die fünfseitige „Auswahlbibliographie“ gerechtfertigt, in der die vom Autor konsultierte Literatur aufgelistet ist: vor allem Biographien (38 Titel) und kunst- bzw. kulturhistorische Werke (25), aber auch Briefwechsel (12), Tagebücher (7) und Autobiographien (2). ${ }^{2}$ Illies selbst ist mit einem Magister in Kunstgeschichte fachlich einschlägig vorgebildet und seine bisherigen Buchpublikationen zeigten keine Ambitionen, sich in das Feld der Hochliteratur einzuschreiben, sondern trugen eher journalistisch-essayistischen Charakter, wie sein großer Erfolg Generation Golf von 2000.

Dennoch lässt sich das Buch nicht so einfach im Ordner Sachbuch ablegen. Unklar wäre bereits, welcher Sachbereich denn hier eigentlich behandelt wird: Geschichte? Das wird durch die Wahl des Ausschnitts - ein spezifisches Jahr und die Gliederung in 12 Monatskapitel nahegelegt, aber von den entscheidenden historischen

\footnotetext{
${ }^{2}$ Hier erscheinen auch Gumbrechts 1926 und weitere Anregungen für die spezifische Form wie Frederic Mortons (1924 in Wien als Fritz Mandelbaum geboren)1990 publizierter historische Roman Thunder at Twilight: Vienna 1913/1914. In verblüffend ähnlicher Weise stellt das erste Kapitel von Morton die Figuren Stalin, Kaiser Franz Joseph und Hitler nebeneinander. Auch hier wird Stalin bei der Ankunft mit Koffer und derben Schuhen dargestellt, sein großer Schnurrbart kann die Pockennarben kaum verdecken, er hat Lenin im Schach geschlagen und ist schlecht im Radfahren. (MORTON 1990: 5 f.; ILliES 2012: 11 f.) Das mögen zwar „historische Tatsachen“ sein, die auch in den einschlägigen Biographien vorkommen, aber die Dichte der parallelen Nennungen ist auffällig.
} 
Galle, H. -Geschichtsdarstellung in der Gegenwartsliteratur

Ereignissen dieses Jahres (Balkankrieg: 182; Rüstungsausgaben in Europa: 108; Erhöhung der Truppenstärke in Deutschland von 117.267 auf 661.478 Mann: 159) ${ }^{3}$ erfährt man bei der Lektüre nur sehr beiläufig. Kunstgeschichte? In der Tat gilt den bildenden Künstlern von Picasso bis Kokoschka das Hauptinteresse, aber es fehlen weitgehend die sachlichen Kommentare $\mathrm{zu}$ ihrer Kunst. Am ehesten also Kulturgeschichte, denn es gibt immer wieder Zitate aus populären Medien wie der Gartenlaube und dem Simplicissimus, die sich mit Mode (Schlankheit: 79 f.), Sport (Tennis: 120), Werbung (Drogistenkalender: 103), Erfindungen (Droge Ecstasy: 18) und technischen Neuerungen (Gasbeleuchtung: 45, Kampfflugzeuge: 104, Wolkenkratzer: 111, Funkentelegraphie: 120, Fließbandarbeit bei Ford: 200) befassen. Doch fehlen auch hier erklärende Texte, die solche episodischen Nennungen in den historischen Zusammenhang einordnen. Indem es die „große Erzählung“ verweigert, nähert sich das Buch den Versuchen von Kempowski und Gumbrecht. Wie WEBER (2012) betont, ermöglicht auch 1913 einen vom Wissen der Nachwelt befreiten Blick auf die Ereignisse vor Ausbruch des Weltkrieges und ihre Wahrnehmung durch die Zeitgenossen. Im Unterschied zu Kempowski jedoch lässt es nicht ausschließlich Dokumente sprechen, sondern gibt deren Inhalt in Form von „kleinen Erzählungen“ wieder, die - anders als bei Gumbrecht - keinen Anspruch auf wissenschaftliche Genauigkeit erheben, aber unbestreitbaren Unterhaltungswert besitzen.

Es ist die erste Sekunde des Jahres 1913. Ein Schuss hallt durch die dunkle Nacht. Man hört ein kurzes Klicken, die Finger am Abzug spannen sich an, dann ein zweiter, dumpfer Schuss. Die alarmierte Polizei eilt herbei und nimmt den Schützen sofort fest. Er heißt Louis Armstrong.

Mit einem gestohlenen Revolver hatte der Zwölfjährige in New Orleans das neue Jahr begrüßen wollen. Die Polizei steckt ihn in eine Zelle und schickt ihn schon am frühen Morgen des 1. Januar in eine Besserungsanstalt, das Colored Waifs' ${ }^{`}$ Home for Boys. Er führt sich dort so wild auf, dass der Leiter der Anstalt, Peter Davis, sich nicht anders zu helfen weiß, als ihm spontan eine Trompete in die Hand zu drücken (eigentlich hatte er ihn ohrfeigen wollen). Louis Armstrong aber wird urplötzlich stumm, nimmt das Instrument fast zärtlich entgegen, und seine Finger, die zuvor nervös mit dem Abzug des Revolvers gespielt hatten, spüren erneut das kalte Metall, doch statt eines Schusses entlockt er der Trompete noch im Zimmer des Direktors erste warme, wilde Töne. (9)

So beginnt das Buch mit einer Szene, die zwar also solche dokumentiert ist, aber in ihren Einzelheiten mit Mitteln ausformuliert ist, die sich kaum von fiktionaler Prosa unterscheiden. Die Grunddaten mögen zutreffen (der Pistolenschuss und das Heim

\footnotetext{
${ }^{3}$ Im Folgenden werden für alle Zitate aus Illies` Buch lediglich die Seitenzahlen angegeben.
} 
Galle, H. -Geschichtsdarstellung in der Gegenwartsliteratur

finden sich im Wikipedia-Artikel zu Armstrong), aber die Gestaltung ist alles andere als historisches Berichten. Vielmehr wird die Aufmerksamkeit des Lesers wie in einer Filmsequenz geführt. Dies wird unterstützt durch das Präsens, in dem das ganze Buch gehalten ist. Dass der Leiter der Anstalt den Knaben „hatte ohrfeigen wollen“ mag er irgendwann einmal zu Protokoll gegeben haben, aber als Leser erhält man diese Auskunft von einem auktorialen Erzähler, der ebenso weiß, was der kleine Armstrong „spürt“, als er zum ersten Mal die Trompete (in Wirklichkeit wohl eher ein Kornett, das Armstrong schon zwei Jahre zuvor zu spielen gelernt hatte) in die Hand nimmt, um ihm „erste warme, wilde Töne“ zu entlocken, eine Beschreibung, die sich sicherlich eher den späteren Schallplattenaufnahmen verdankt, als den Wahrnehmungen der Beteiligten am 1. Januar 1913. Diese Schreibweise geht mit der Kulturgeschichte ähnlich um, wie der New Journalism seit den 60er Jahren und die Non-fiction Novel Truman Capotes, der sich anheischig machte, die Morde von Holcomb (In Cold Blood, 1965) so zu beschreiben, dass nichts anderes als die bekannten Fakten, aber in durchaus romanhafter Form dargestellt würde. Im Sinne Kendall WALTONS (1990: 79) wäre durchaus ein fiktionaler Text denkbar, in dem jeder einzelne Satz vom Autor als wahrer Satz reklamiert würde. Capote und Illies bewegen sich in diesem Grenzbereich von nicht erfundenen, aber ästhetisch fiktionalisierten Szenen.

$\mathrm{Zu}$ dieser fiktionalen Ausgestaltung der Einzelszene kommt hinzu, dass die Anekdote symbolisch aufgeladen ist: Der heranwachsende schwarze Underdog lässt die Waffe und greift zur Trompete, um für den Rest seines Lebens die Menschheit mit seiner Kunst zu beglücken. Der Amerikaner tut also genau das Gegenteil von dem, was die Europäer ein Jahr später tun werden, wenn sie ihre Pinsel und Schreibfedern mit Gewehren vertauschen und in die Vernichtungsorgie des 20. Jahrhunderts stürmen. Kein Zweifel, dass Illies die Episode absichtsvoll so komponiert hat, um an den Beginn seines Hymnus auf den ungeheuren kulturellen Reichtum Europas vor der Katastrophe das zu stellen, was er für die große Alternative zum desaströsen Gang der Geschichte hält: Hätte man sich damals einfach weiter exklusiv der Kunst und dem Lebensgenuss gewidmet, wäre der Menschheit das ganze Grauen erspart geblieben.

Dadurch mag deutlich werden, dass 1913 bestimmte Techniken der fiktionalen Literatur adaptiert, zu denen auch der durchgehende Gebrauch des Präsens und die erlebte Rede gehören. Auch wenn zentrale Eigenschaften der Fiktion fehlen - die Einführung fiktiver Figuren und Handlungen - schafft das imaginative Ausmalen der 
Galle, H. -Geschichtsdarstellung in der Gegenwartsliteratur

dokumentierten Situationen, wie sich schon in der zitierten ersten Szene zeigt, eine fiktionsähnliche Textstruktur, die dem Leser das Eintauchen in die erzählte Welt ermöglicht. Es ist also durchaus sinnvoll, es im Zusammenhang mit literarischen Darstellungen von Geschichte zu analysieren. Der große Erfolg bei Lesern und Kritik die wichtigen Printmedien (Matussek 2012; Pfohlmann 2012; Seibt 2012; Trotha 2012; Wallasch 2012; Weber 2012; Kluge 2013) haben es, soweit ich sehe, durchweg sehr positiv rezensiert - spricht auch dafür, dass hier zumindest sehr geschickt gearbeitet wurde, um eine affektive Leseerfahrung mit historischen Informationen zu verbinden. Das Buch ist, wie man sagen würde, „süffig“ und der anspruchsvollere Leser stößt sich möglicherweise zunächst nur an der überflüssigen Nennung der Namen von „alten Bekannten“, die vom halbgebildeten Leser ohnehin schon identifiziert wurden, sowie an der schnodderigen Ausdrucksweise und der Schlüssellochperspektive der meisten Geschichten. Aber gerade diese Eigenschaften dürften andererseits zum Verkaufserfolg von diesem ,,angenehm untheoretischen Buch“ beigetragen haben, über das sich Alexander WALLASCH (2012) in der taz freut: „Ein lustiges Durchatmen also nach jedem dritten oder vierten Absatz Hochkultur.“ Keine Frage, die dargestellten Personen gehören größtenteils zum Höhenkamm von Klassischer Moderne und Avantgarde, also eigentlich „schwerer Kost“. Aber wo würde der Text von Illies selbst zu „Hochkultur“, zu einem Kunstwerk, das so hohe Ansprüche an den Leser stellt, dass er von Zeit zu Zeit „durchatmen“ müsste? Im Folgenden soll dieser Frage und der spezifischen Art von Hybridität von 1913 mit einem Blick auf die narrative Struktur und die dargestellten Inhalte genauer untersucht werden.

\section{Erzählstruktur: Collage und Perspektivieruns}

Da sind zunächst die Erzählstruktur und der Erzähler. Es wurde bereits angedeutet, dass er durch seine respektlose Nähe zu den Gedanken und Gefühlen der Figuren als tendenziell auktorialer Erzähler ausgewiesen ist. Doch andererseits präsentiert sich der Text als Sachbuch und nichts deutet explizit auf eine bewusste Trennung von Autor und Erzähler hin. Da ausschließlich historische Personen und Ereignisse behandelt werden, muss der Leser schließen, dass hier Wirklichkeitsaussagen gemacht werden, die der Autor selbst verantwortet, gestützt auf die von ihm studierte Sekundärliteratur. 
Entsprechend können ihm eventuelle Fehler im Umgang mit der historischen Wahrheit angerechnet werden (ansatzweise: KEUSCHNIG 2013), denn sie sind nicht durch die Lizenz der Fiktion gedeckt.

Wie lässt sich der Widerspruch zwischen fiktionaler Schreibweise und nichtfiktionalem Inhalt lösen? Der Autor könnte seine Aussagen über die Gedanken und Gefühle der Protagonisten dadurch rechtfertigen, dass sie mit einer gewissen Logik aus dem vorhandenen Datenmaterial hervorgehen. An manchen Stellen fügt er noch ein entschuldigendes ,ganz genau wissen wir es nicht“ (11) oder „so stellt man sich das jedenfalls vor“ (306) ein. Überhaupt findet sich das Pronomen „man“ sehr häufig und eröffnet das Selbstverständnis dieses Erzählers: er steht stellvertretend für das von ihm imaginierte Kollektiv seiner Leser, für das zeitgenössische Publikum. Manchmal wechselt er sinnfällig zum „wir“: „Und wir wissen es auch [dass August Mackes Bilder aus Tunis Kunst sind]. Es sind Bilder von solch echter bezwingender Schönheit, dass man sie manchmal nur ertragen kann, wenn man versucht, sie als Kitsch zu denunzieren.“ (259). Der studierte Kunsthistoriker und emphatische Bewunderer der Avantgarde fühlt sich offenbar im Recht, wenn er dieses ästhetische Urteil stellvertretend für den Mainstream seiner Leser abgibt.

Mit seinem Publikum plaudert er auch ganz vertraulich und ungekünstelt, indem er immer wieder ein „übrigens“, „,apropos“, „,eigentlich“, „unter uns“ oder auch „aber das verwirrt jetzt nur“ einflicht, ganz im Stil der informellen Konversation. Er ist weniger Historiker als vielmehr Conférencier, der weder literarisch noch inhaltlich hochkulturelle Ansprüche stellt und seinem Publikum plaudernd und augenzwinkernd seine Funde vorführt. Das „dominante Archiv“, wie Moritz BAßLER (2005: 35) vielleicht sagen würde, sind nicht die vorgeführten Protagonisten von 1913, sondern der Kunst- und Geschichtskonsument der Gegenwart, sein Geschmack, sein Wissen, seine Wertvorstellungen und seine Interessen.

Seine Perspektive auf das Vergangene ist von demselben informellen Umgang geprägt. Die ersten Episoden werden in der Manier des auktorialen Erzählers unmittelbar eingeführt, der als unsichtbarer Beobachter überall präsent sein kann. Bestimmte Personen werden wie alte Freunde behandelt: „Apropos kränkelnd. Wo steckt eigentlich Rilke?“ (13); „Und wie geht es Ernst Jünger?“ (18). Diese Fragen können aufgrund der dokumentierten Biographien scheinbar beantwortet werden und genau dies tut der Erzähler, indem er dem Leser auf einer fiktionalen Ebene die Illusion 
Galle, H. -Geschichtsdarstellung in der Gegenwartsliteratur

der Präsenz der recherchierten Realität verschafft, zwischen der er hin- und herschaltet wie ein Fernsehmoderator zwischen den Brennpunkten des live berichteten Geschehens: „Da meldet sich endlich Rainer Maria Rilke!“ (27); „Wir dürfen Kafka nicht vergessen und seine Braut!“ (207); „Noch einmal kurz zu Freud in die Berggasse 19.“ (32). So entsteht der Eindruck einer vergangenen Realität, die unserem beobachtenden Zugriff weitgehend zugänglich ist, ohne dass große Zweifel an der Faktizität dieser auf Dokumente gestützten Rekonstruktion aufkommen müssten.

Auf diese Weise entstehen auch die narrativen Verknüpfungen zwischen den Episoden, die ja nur gemeinsam haben, dass sie (mehr oder weniger) gleichzeitig in den europäischen Metropolen Berlin, Wien und Paris geschehen. Denn an sich sind diese Ereignisse „kontingent“ im ursprünglichen Sinn des Wortes: sie stoßen in Raum und Zeit aneinander, ohne logisch oder kausal auseinander hervorzugehen. Nur einige der Figuren standen tatsächlich in Kontakt miteinander, die meisten wussten gar nichts von der Existenz der anderen Akteure, mit denen sie hier zusammengeführt werden.

Die Komposition des Buches ermöglicht dem Leser (wie Kempowskis Echolot) eine „horizontale“ und eine ,vertikale“ Lektüre. Innerhalb des Kapitels zum Monat Januar nehmen wir bei der horizontalen Rezeption das gleichzeitige Miteinander von unterschiedlichen Personen und Phänomenen wahr (in dieser Reihenfolge): Armstrong, Kafka, Stalin, Freud, Schönberg, Th. Mann, Kraus, Lasker-Schüler, Nofretete (die Büste der Pharaonin), Lutz (der Eiskunstläufer), Stalin, Ecstasy, Rilke, Spengler, Fotos von der Titanic, Kafka, Picasso und Braque, Corinth, Freud und Jung, Adorno, Proust, G. Stein, Beckmann. Aber die „Geschichten“ über Kafka, Freud, Kraus und Rilke u. v. a. lassen sich auch vertikal durch die zwölf Kapitel hindurch verfolgen und kommen dabei sogar unter Umständen zu einem Abschluss wie die Amour fou Kokoschkas zu Alma Mahler oder die Geschichte von Louis Armstrong, über dessen erstem Auftritt im September 1913 gesagt wird, er höre „,nie wieder auf“ zu spielen und würde „zum größten Jazz-Trompeter der Geschichte“ (224).

Auch eine andere Geschichte wird von Illies benutzt, um zwischen den Monaten Kohärenz herzustellen und die Folge unzusammenhängender Ereignisse zu einer Closure zu bringen: der Diebstahl der Mona Lisa aus dem Louvre. Dieser lag zwar selbst schon zwei Jahre zurück, aber seine Aufklärung fiel in den Dezember 1913, und Illies fügt in den vorangehenden Monaten jeweils Hinweise auf den ungeklärten Stand der Dinge ein, die - wie viele ähnliche Elemente - als Leitmotiv fungieren. Da Picasso 
Galle, H. -Geschichtsdarstellung in der Gegenwartsliteratur

ursprünglich einer der Verdächtigen war und die Gioconda gerade durch diesen Diebstahl zur Ikone der Malerei schlechthin avancierte, rundet dieser Fall die Einblicke in den damaligen Kunstbetrieb mit einem Ausblick auf unser heutiges, massenmedial geprägtes Verhältnis zur Kunst und gibt dem Buch zugleich eine Kohärenz stiftende Note von Kriminalroman.

Ähnlich salopp wie sich der Erzähler dem Leser als Konversationspartner anbietet, verhält er sich gegenüber den Objekten seiner Erzählung. Bestimmte Personen werden nicht nur wie vertraute Freunde behandelt, sondern auch mit einer mitleidigen Herablassung. Die Hypochondrie und Menschenscheu von Rilke, Kafka und Spengler, die Eitelkeit Thomas Manns erscheinen im ironischen Diskurs des Erzählers einigermaßen lächerlich, weil die Anlässe ihrer Probleme schon damals für Außenstehende irrelevant waren und sich aus heutiger, welthistorischer Sicht völlig relativiert haben.

In Prag leidet Kafka. Darunter, dass seine aus der Ferne angeschmachtete Felice nichts sagt zu dem Band ,Betrachtungen“, den er ihr im Dezember geschickt hatte. Darunter, dass seine Schwester Valli heiratet, darunter, dass es in der Wohnung immer so laut ist (weil die Türen klappen und seine Eltern und seine Schwestern zu reden wagen), darunter, dass er tagsüber in der Versicherung arbeitet und nachts an seinem Werk. (50).

Wenn man weiß, wie ausweglos Kafka tatsächlich existentiell unter seiner Situation gelitten hat, wie sehr er dieses sein Leiden für absurd hielt und welche Werke daraus hervorgegangen sind, dann vergeht der Witz. Aber aus der Distanz von 100 Jahren mag eine gewisse Heiterkeit angesichts des Autorenlebens walten, wenn denn das Werk mit seinem ganzen Ernst dagegen steht. Aussagen wie „Rainer Maria Rilke hat Schnupfen $(85)^{\text {“ }}$ bleiben denunziatorisch, denn sie verschweigen, dass die extreme Sensibilität des Dichters in derselben Zeit den ersten Anlauf zu den Duineser Elegien nennt; Rilkes autobiographischer Text „Das Testament“ vermittelt eine Vorstellung von der extremen Konzentration, die es brauchte, um dieses Werk schließlich zu abzuschließen. Was von der Kunst dieser Autoren in Illies“ Text erscheint, ist eher spärlich und bildet kein Gegengewicht zu den banal erscheinenden Nöten dieser verschnupften Hypochonder, Neurastheniker. Übrigens: der banale Schnupfen taucht auch im emblematischen Gedicht „Weltende“ (1911) von Jakob van Hoddis (eigentlich: Hans Davidsohn) auf. Scheinbar harmlos reiht sich die Aussage „Die meisten Menschen haben einen Schnupfen“ in die parataktische Folge von Katastrophen ein, von denen die Zivilisation 
Galle, H. -Geschichtsdarstellung in der Gegenwartsliteratur

bedroht ist. Und doch ist der banale Schnupfen wie alle anderen Phänomene des „Weltendes“ ein äußerer Eingriff in die Integrität des Menschen, dem er hilflos ausgeliefert ist, auch wenn er - meist - ohne Folgen bleibt. Aber nicht nur im Verhältnis zum Werk, auch im Hinblick auf Rilkes vorzeitigen Tod an Leukämie und die Tuberkulose Kafkas wirkt der Spott über die Überempfindlichkeit der Autoren vor allem geschmacklos und billig.

Diese Art der Behandlung der Protagonisten ähnelt jedoch stark dem ironischen Verfahren Kehlmanns in Die Vermessung der Welt. Auch hier kann sich der Leser schmunzelnd als Komplize des Erzählers fühlen und sich über die Schwächen der Geistesheroen erheitern und zugleich das erhebende Gefühl verspüren, großen Momenten der Geschichte beizuwohnen. Anstelle einer Illusionsbrechung durch Selbstreferenzialität wie in den historischen Romanen der Postmoderne, kommt es hier eher zu einer Verstärkung der Illusion, zumal bei Illies, der ja nicht als Roman daherkommt. Die ironische Perspektivierung wird den Figuren sicher in vielen Fällen nicht gerecht, aber erlaubt es, sehr nahe an die Personen heranzukommen und sie ungestraft mit der Respektlosigkeit und Überlegenheit des Erzählers zu beurteilen, als wären sie merkwürdige Typen aus unserem Bekanntenkreis.

\section{Inhalt: große Kunst und kleine Künstler}

Damit kommen wir zu den eigentlichen Inhalten von 1913. Die Kunst - besser gesagt die Künstler und sonstige Akteure des Kulturbetriebes - stehen qualitativ und quantitativ im Zentrum des Interesses. Daneben gibt es aber auch einzelne Hinweise auf die Politik. Da sind zum einen die Machthaber - Kaiser Franz Joseph und der österreichische Thronfolger Franz Ferdinand sowie der deutsche Kaiser Wilhelm II deren Aktivitäten sich hier vor allem in Ausfahrten per Kutsche und Jagden (210, 244, 282, 286) zu erschöpfen scheinen. Über die tatsächliche Politik der beiden Reiche erfährt man vor allem durch Hinweise auf die Erhöhung der Truppenstärke $(159,198)$. Diese lakonischen aber scheinbar eindeutigen Hinweise auf den kommenden Krieg stehen in heftigem Kontrast sowohl zu dem Benehmen der Herrscher, zur unbekümmerten Geschäftigkeit des kulturellen Lebens und den zitierten Aussagen über die Unwahrscheinlichkeit, ja Unmöglichkeit eines Krieges. Die Widersprüche werden 
nicht thematisiert, der Leser muss sich seinen Reim darauf machen und darf vermuten, dass es Anzeichen gab, welche die Menschen nicht zur Kenntnis nehmen wollten.

Dazu kommen Szenen, in denen die Protagonisten der kommenden Katastrophe beleuchtet werden, vor allem Stalin und Hitler. Der historische Zufall will es, dass diese beiden ebenso wie Trotzki und Tito in jenem Jahr in Wien waren und sich zeitweise begegnet sein könnten. Ganz nahe am Palais Schönbrunn, wo der Kaiser gerade seine Kutsche verlassen hat, begegnen sich bei Illies Stalin und Hitler:

Stalin geht durch den Park, denkt nach, es dämmert schon. Da kommt ihm ein anderer Spaziergänger entgegen, 23 Jahre alt, ein gescheiterter Maler, dem die Akademie die Aufnahme verweigerte und der nun die Zeit totschlägt im Männerwohnheim in der Meldemannstraße. Er wartet, wie Stalin, auf seine große Chance. Sein Name ist Adolf Hitler. Vielleicht haben sich die beiden, von denen ihre Bekannten aus dieser Zeit erzählten, dass sie beide gerne im Park von Schönbrunn spazieren gingen, einmal höflich gegrüßt und den Hut gelüpft, als sie ihre Bahnen zogen durch den unendlichen Park. (25 f.)

An imaginären Koinzidenzen wie dieser leidet das Buch keinen Mangel, auch Kafka, Musil und Joyce hätten sich in Triest treffen können (229) und die Briefe an Felice könnten „im selben Postsack von Prag nach Berlin“ gereist sein wie die „brieflichen Wehklagen Einsteins“ an seine Braut (79). Aber es sind nicht mehr als pittoreske, verblüffende Bilder, die ebenfalls aus einem Film stammen könnten. Dass diese beiden Hauptakteure der Massenvernichtung sich in Wien begegnet sein könnten, wird nur für die Behauptung genutzt, das ,Zeitalter der Extreme, das schreckliche kurze 20. Jahrhundert" beginne an diesem Januarnachmittag des Jahres 1913 in Wien (27). Warum das so sein soll, bleibt unklar, denn nur wenige Seiten später kommentiert der Erzähler die Präsenz von Stalin, Hitler und Tito in Wien so: „Drei Statisten, so möchte man meinen, ohne eigenen Text im großen Schauspiel von ,Wien um 1913‘““(41). Besonders krass wird der Kontrast zwischen diesen „Versagern“ und den wahren Akteuren dieses Jahres im Kapitel November. Mitten unter den sich überschlagenden Nachrichten von den Triumphen der Modernen steht der Absatz: „Am 7. November malt Adolf Hitler ein Aquarell der Münchner Theatinerkirche und verkauft es an einen Trödelhändler auf dem Viktualienmarkt.“ (263). Das ist auf den Punkt gebracht: Was kann man von einem Mann erwarten, der seine konventionellen Veduten in diesem Moment, wo sich der Malerei alle Möglichkeiten eröffnen, für ein paar Mark feilbietet. 
Galle, H. -Geschichtsdarstellung in der Gegenwartsliteratur

So entsteht der Eindruck, als gäbe es eine Opposition zwischen moderner Kunst und totalitärer Politik. Unter den beiden Tyrannen wird die Moderne keine Chance mehr haben, aber als die Kunst blühte, waren diese beiden nur „Statisten“. Dass dies den komplexen Zusammenhängen zwischen den politischen und den künstlerischen Avantgarden nicht gerecht wird (vgl. BECKER / KIESEL 2007), ist dem Autor sicherlich klar. Aber er hat ja auch lediglich die synchronen Ereignisse eines Jahres nebeneinandergestellt, die der Leser mit seinem Wissen verbinden und daraus Schlüsse ziehen kann. Dennoch scheint die Opposition von (moderner) Kunst und einer kleinbürgerlichen Ästhetik das Buch thematisch zu strukturieren, der Postkartenästhetik des Kopisten Hitler, die während seiner Herrschaft nicht nur die offizielle Kunst des Dritten Reichs bestimmen, sondern integraler Bestandteil der Ideologie, der Machtausübung und der Inszenierung der Zerstörung sein wird. In 1913 entspricht dieser Gegensatz „Krieg“ zwischen konventionellem Geschmack und moderner Kunst: „Berlin, Paris, München, Wien: Das waren die vier Frontstädte der Moderne 1913.“ (42). Zwar werden auch zwischen den Künstlern ,außenpolitische Scharmützel““ ausgetragen, doch über allem gibt es eine ,ästhetische Allianz über Grenzen hinweg, eine Demonstration der Zusammengehörigkeit der Avantgarde“ (233).

Die große Auseinandersetzung findet statt zwischen der Kunst und dem unverständigen zeitgenössischen Publikum bzw. der konservativen Kritik und Illies ergreift eindeutig Partei für die Modernen mit Aussagen wie „Das [die Einstufung eines Künstlers als Primitivling durch das amerikanische Publikum] ist wie stets langfristig der größte Qualitätsbeweis.“ (49) oder „Die deutsche Kritik ätzte weiter.“(246). Seine Favoriten sind die Pariser Künstler um Picasso, Braque und Duchamp sowie die deutschen Expressionisten der Brücke und des Blauen Reiters, daneben aber auch Einzelgänger wie Lovis Corinth und Beckmann. Komponisten wie Schönberg und Strawinsky, Literaten wie Schnitzler, Kafka, Rilke, Lasker-Schüler und Benn stehen an derselben „Front“ und kämpfen für „das Neue“. Die im Deutschen eingebürgerte Bezeichnung „Moderne“ ist dem Autor jedoch nicht ganz geheuer:

Ohnehin muss jetzt mal Schluss sein mit der ,Moderne“ in diesem Jahr - das ist ein solch flexibler Begriff, von Zeitgenossen und Nachgeborenen immer wieder anders ausgelegt und von jeder Generation zeitlich wieder neu angesiedelt, dass er eigentlich gar nicht mehr taugt, um die ungeheure ungleichzeitige Gleichzeitigkeit, die das Jahr 1913 vor allem ausmacht, angemessen zu schildern. (76) 
Zwar bezieht sich das zunächst auf den gerade erfolgenden Übergang von der impressionistischen Avantgarde zu denen des 20. Jahrhunderts, dem der Kritiker MeyerGräfe nicht mehr zu folgen vermag, aber aus Illies` Äußerung wird klar, dass er den Moderne-Begriff eigentlich verabschieden möchte, um ihn in einem emphatischen, umfassenden Begriff von Kunst aufgehen zu lassen. Wenn immer wieder von einer neuen Revolution die Rede ist - der kubistischen, der expressionistischen, der des Ready-mades - so werden diese Umwälzungen der Ästhetik nicht aus einer inneren Logik der Kunst, des Marktes oder der Modernisierung erklärt, sondern den jeweiligen Künstlern als persönliches Verdienst zugeschrieben. So sind die Auseinandersetzungen der Künstler und ihrer Propagandisten mit der konservativen Öffentlichkeit ein zentrales Thema, aber der Akzent des ganzen Buches liegt eigentlich auf den existentiellen Befindlichkeiten der Personen. Bei Oswald Spengler (und Kafka) ist es die „Angst vor Weibern, sobald sie sich ausziehen“ $(29,44)$, bei Freud der „Vater-Sohn-Konflikt“ mit Carl Gustav Jung (32), bei Rilke die Hypochondrie, bei Else Lasker-Schüler das Liebesleid usw.

Vor allem aber sind es immer wieder die Schlafzimmergeschichten, die als Inhalt und Schlüssel zu den Ereignissen fungieren. Dass Stalin, anders als Bucharin, keinen Erfolg beim Wiener Zimmermädchen hatte, ist offenbar der Grund für den späteren Mord an dem Genossen (68). Ebenso wird Alfred Kerrs Rachsucht, ,aber das muss unter uns bleiben“, zum Motiv für seinen Verriss von Thomas Manns Stück Fiorenza, denn dieser hatte ihm schließlich einst die umworbene Katja Pringsheim weggeschnappt, „die reiche Jüdin mit den Katzenaugen“(15). Kokoschkas sexuelle Obsession und seine Abhängigkeit von Alma Mahler bringen das Meisterwerk „Die Windsbraut" hervor. Picasso muss mit seiner neuen Geliebten Eva vor den Zudringlichkeiten der Ex-Geliebten Fernande fliehen (204). Aus der unglücklichen Ehe Hermann Hesses entsteht der Roman Roßhalde (65). Rilke gelingt neben einem Liebesgedicht endlich eine „halbwegs unkomplizierte Affäre“ (197). Heinrich Mann fertigt Zeichnungen an: „meist wohlbeleibte Frauen in gewagten Posen“ (63). Kafka stürzt sich „für zehn Tage in eine kindliche Verliebtheit [...], die zu nichts führen muss.“ (231) Karl Kraus findet endlich zu Sidonie Nádherný, der Liebe seines Lebens (306 f.). Carl Schmitt wartet auf seine Entdeckung, während seine Geliebte Cari nachts „herrlich unartig“ ist (235) usw. usf. Diese Schlüssellochperspektive auf die Protagonisten ist so unterhaltsam wie der Klatsch auf einer Party, dient aber nicht etwa 
Galle, H. -Geschichtsdarstellung in der Gegenwartsliteratur

dazu, vermeintlich Helden und Genies auf ihre menschlichen Dimensionen zurückzuführen, denn Genie bleibt bei Illies durch und durch Genie: „Dr. Alfred Döblin, großer Arzt, großer Autor und großer Freund Ernst Ludwig Kirchners [schreibt in seinem Brief an Marinetti] die herrlichen Worte [...]“ (242). Kerniger hätte auch Homer die Epitheta für seinen Helden Odysseus nicht wählen können.

Das Problem an der Verengung der Perspektive auf die Personen und ihre vor allem erotischen Nöte liegt, wie bereits erwähnt, darin, dass die eigentliche Wirkung der Kunst sich im Roman nicht manifestiert. Im Wesentlichen ist die Präsenz der Kunst selbst im Buch durch blasse Schwarz-Weiß-Reproduktionen zu Beginn jedes Kapitels gewährleistet und durch einige wenige wörtliche Zitate aus Gedichten von Rilke, Brecht, Trakl und Tucholsky. Wo es darum ginge, die Literatur als eigene Dimension erfahrbar zu machen, wird ihre Wirkung vor allem behauptet und ihre Stärke aus der Intensität der existentiellen Erfahrung des Künstlers abgeleitet. So heißt es von den Morgue-Gedichten Gottfried Benns: „Schonungslose, kalte und doch verwegen spätromantische Gedichte über Körper, Krebs und Blut, die eine große, existentielle Erschütterung verraten und die man auf nüchternen Magen noch heute nicht lesen kann.“(59) Auch Trakls Verse sind „,zu existentiell gelebt [...], als dass man sie des Wortrausches zeihen könnte, des Kitsches gar.“(274).

Trotz aller vom Autor behaupteten revolutionären Brüche mit der romantischen Kunst des 19. Jahrhunderts (Gab es keinen Realismus? Gab es keine Frühromantik?) scheint er selbst ihre Essenz in der Mimese zu erkennen, selbst in der Musik: „Und während er [Armstrong] allein auf seinem Bett sitzt und eine Fliege durch das Zimmer fliegt, versucht er ihren Flug mit seinen Tönen nachzuahmen, er folgt ihr nach, brummend, stoppend, brummend.“ (224) Ebenso sieht Illies die expressionistischen Ausdrucksformen Kirchners vor dem Paradigma der Abbildung:

Auf kleinstem Raum verdichtet, ist hier die städtische Moderne zu sehen, die Großstadt, und ihre Hauptdarsteller, die Kokotten in ihren schrillen Farben und mit ihren toten Gesichtern, die den Männern ein Glück verheißen, an das nicht einmal der Freier mehr glauben kann. Kirchner spürt, wie die Körperlichkeit, die er in Fehmarn an den Frauen und den Kindern noch als pure Natürlichkeit hat erleben und malen können, im Stadtraum der Neuzeit, unter den Gewändern, dem Lärm, unter den anderen Blicken und den anderen Erwartungen, nicht mehr möglich ist. (248).

Modern malen kann man offenbar erst, wenn man sie Sujets einer modernen Großstadt vor sich hat. Betrachtet man jedoch die hier erwähnten Bilder von der Insel Fehmarn, 
Galle, H. -Geschichtsdarstellung in der Gegenwartsliteratur

wird diese Differenz fraglich, denn Kirchner hat auch hier offenbar schon dieselben Abstraktionen vorgenommen, die Illies als Qualitäten den entfremdeten Großstadtmenschen zuschreibt.

Wo der Fokus auf den Künstlern und ihren alltäglichen Verrichtungen liegt, kann die Kunst als solche nicht in Erscheinung treten und es bleibt bei der Evokation des Äußerlichen: Künstler bei der Arbeit. ,Jetzt schreibt er [Musil] den schönen Satz: ,Ulrich sagte das Schicksal vorher und hatte davon keine Ahnung. ' Nicht schlecht.“ (306). Zweifellos hat es der Satz von Musil in sich, vor allem wenn er auf all die Äußerungen der Akteure von 1913 bezogen wird, die im Nachhinein als prophetisch verstanden werden könnten. Freilich schrieb Musil den Satz nicht im „Jetzt“ des Jahres 1913, sondern erst viel später, als er, um vieles weiser, seinen Protagonisten und die ganze Parallelaktion in dieses ominöse letzte Jahr vor dem Ausbruch des Krieges versetzte.

Zum Abschluss seines Amour de Swann „schreibt [Proust] mit feiner Tinte den letzten Satz aufs Papier“ (35). Letzte Sätze und erste Sätze von großen Romanen werden auf die Situation der Menschen in diesem Jahr hin gedeutet und funktionieren gewissermaßen wie Markenzeichen, ihre Nennung erübrigt ein tieferes Eindringen in das Werk. „,[...] Es war ein schöner Augusttag des Jahres 1913.‘ Mit diesen Worten lässt Robert Musil seinen ,Mann ohne Eigenschaften` beginnen. Neben Prousts ,Recherche“ und James Joyce ,Ulysses“ der dritte Klassiker der Moderne, in dem die Sprengkraft des Jahres 1913 aufgesogen ist.“ (199). Es bleibt rätselhaft, wie diese Sprengkraft in die Texte gelangt sein mag: die Arbeit Prousts an seinem Werk erstreckte sich über vierzehn Jahre, 1913 markiert nur die Publikation des ersten Teil, Joyce begann gerade erst, den Ulysses zu konzipieren, und Musil verlegt die Handlung in jenes Jahr, wohl eher weil es das letzte ruhige Jahr vor dem Krieg war, als wegen seiner besonderen Sprengkraft.

Die Größe des Kunstwerks wird vor allem rhetorisch behauptet, indem die gängigen Urteile der Kunstgeschichte wiederholt werden. So gelingt Marcel Duchamp mit seinem ready-made „der beiläufigste Paradigmenwechsel der Kunstgeschichte“ (284) und „Während sich also in Paris gerade das erste ready-made, das Vorderrad auf dem Schemel, in der Hand von Marcel Duchamp dreht, entsteht in Moskau das erste ,Schwarze Quadrat' - die beiden Nullpunkte der modernen Kunst.“ (291). Es scheint, 
als bedürfe das Buch außer den Bettgeschichten vor allem solcher greatest hits, um die Bedeutung der Kunst zu rechtfertigen.

Als Joyce durch Ezra Pound eine Publikationsmöglichkeit für seine Erzählungen und das Porträt des Künstlers erhält, befindet der Erzähler: „A star is born.“ (297). Nicht um eine spezifische ästhetische Wahrnehmung, nicht um eine andere Form der Erkenntnis geht es, sondern um die Produktion von Objekten für den Massenkonsum: Pop. Joyce ist ein Star und daher sind seine Werke hip. Gelesen haben schadet nichts, muss man aber auch nicht unbedingt, um ihn großartig zu finden. Bei den Werken des Expressionismus, bei Picasso und Duchamp ist das nicht grundsätzlich anders. Sie sind heute die Attraktion internationaler Ausstellungen und die Museen moderner Kunst haben sich in den letzten 50 Jahren zu Publikumsmagneten entwickelt, von den Reproduktionen auf Plakaten, Bildschirmschonern und Devotionalien aller Art ganz zu schweigen. Entsprechend ist auch die Debatte um Kitsch oder Kunst, die einem August Macke noch so wichtig war, für Illies hinfällig geworden: die Bilder sind „,von [...] echter bezwingender Schönheit“" (192) und das ist genug.

In diesen Zusammenhang passen auch die in den Text verstreut einfügten Geburtstage von Figuren wie Marika Rökk, Gert Fröbe, Burt Lancaster oder Willy Brandt. Sie gehören in die Populäre Kultur der Kriegs- und Nachkriegszeit, ihre Geburt im Jahr 1913 ist ohne jede Relevanz für die damaligen Geschehnisse. Gleiches gilt für die Gründung des Modehauses Prada oder die Erfindung von Ecstasy, die erst Jahrzehnte später bedeutsam werden. Der Leser, immer ,auf Augenhöhe“ mit den großen Künstlern und ihren Nöten, kann sicher sein, dass seine Vorstellungen von Kunst und Unterhaltung in keinem Augenblick enttäuscht werden. Am allerwenigsten von der Ästhetik des Buches selbst, denn alles Avantgardistische ist dieser Schreibweise zutiefst fremd.

So wie die Gioconda und die Nofretete zu tausendfach reproduzierten Ikonen von Kunst geworden sind, deren Geltung Jahr für Jahr von Millionen Touristen beglaubigt wird, so stellen die Werke der einstigen Avantgarden durch ihre tausendfache Wiedergabe in keiner Weise mehr Hindernisse für die Rezeption (wie dies bei Gegenwartskunst durchaus der Fall ist, sofern sie sich avantgardistisch äußert). Frei von aller ästhetischen Modernität kann sich der Leser von Illies` Buch doch revolutionär fühlen wie seine Idole der Jahrhundertwende, denn es bricht mit den Standards der elitären Literatur und fragt nicht länger nach Kitsch oder Kunst, wenn es nur „schön“ 
Galle, H. -Geschichtsdarstellung in der Gegenwartsliteratur

ist, d.h. gut unterhält. Schwer nachzuvollziehen ist das Urteil PFOHLMANNs (2012), der in dem Buch just die radikalste Montagetechnik der Avantgarde am Werke sieht: „Nicht zuletzt von Picasso und seinem just in dieser Zeit entwickelten synthetischen Kubismus ist Illies' Jahresquerschnitt «1913 - Der Sommer des Jahrhunderts» inspiriert. Souverän und mit grosser Spielfreude montiert und arrangiert [er] sein Material.“ Aber Picassos spröde Bilder von 1913 („Man with Guitar“, „Violin“, „Head“ etc.) erscheinen vor allem als formale, zur Abstraktion tendierende Experimente, die zwar einzelne fremdartige, aber gerade noch erkennbare Wirklichkeitsfragmente in sich aufnehmen, diese jedoch zugunsten einer in sich stimmigen ästhetischen Gesamtwirkung auflösen, die nichts mehr repräsentiert außer sich selbst. Die Einzelepisoden, aus denen Illies sein Buch montiert hat, sind in sich völlig verständliche, geradezu familiäre Geschichten, die auch im Ganzen ein überaus lesbares Panorama ergeben, das dem Leser keinerlei Anstrengungen abverlangt, sich auf einen neuen Code einzustellen, eine kunstvoll verfremdete Realität neu wahrzunehmen. Es sei denn, man will in den burlesken Karikaturen der Protagonisten eine Form von Verfremdung sehen, was aber problematisch bleibt, denn es handelt sich ja um Stereotypen, die da verwendet werden, also um Formen, die eher Wahrnehmungen bestätigen als in Frage stellen.

Die von den Modernen einst provozierten Widerstände sind heute längst vom Massenpublikum assimiliert, werden aber in der identifikatorischen Darstellung Illies“ wieder heraufbeschworen, als gelte es, von neuem Partei zu ergreifen. Hat die sogenannte Pop-Literatur - von der Illies den legeren Tonfall und die Verweigerung von „Tiefe“ übernimmt - noch vor 20 Jahren Proteste im konservativen Feuilleton ausgelöst, so ist es damit heute vorbei. 1913 stößt auf allgemeines Wohlwollen und kann sich damit kaum als aktuelle Version jenes „Neuen“ gerieren, das stets unverstanden von der Masse zurückgewiesen wird. Wenn Matthias MATUSSEK (2012) in seiner Rezension die rhetorische Frage stellt, ob es sich bei 1913 noch um eine Art von Überschreitung handelt, gibt er sogleich selbst die Antwort: eigentlich nicht mehr.

Darf man in solchen Aperçus Geschichte einfangen? Darf man so unangestrengt flanieren zwischen den Zeitebenen, darf man 1913 zum Feuilleton-Kalauer auf dem Promenadendeck machen? Wenn man den Sound so gut beherrscht: bedingungslos ja.

Der Stil heiligt die Mittel und dieser Stil ist Pop: der „Sound“, der allen eingeht, ohne auf Widerstände zu stoßen. Sound ist ,nicht gerade eine eingeführte textanalytische Kategorie“, wie Moritz BAßLER (2005: 143) treffend bemerkt, aber als Metapher aus der 
DJ-Szene auf die Pop-Literatur übertragen steht er für Fähigkeit, heterogene Stücke (Musik / biographisch-historische Episoden) so zu einer einheitlichen Sequenz zu verschmelzen, dass die Tänzer / Leser keine Brüche verspüren. Matusseks Bemerkung scheint insofern durchaus angemessen, als Illies ${ }^{6}$ Schreibweise tatsächlich so unterschiedliche Gegenstände wie Stalin, Kafka und die Mona Lisa zu einer syntaktischen Sequenz amalgamiert, die so eingängig ist wie eine gescheite PartyUnterhaltung. Der kalte Atem, der aus Kafkas Romanen weht, kann so vielleicht benannt oder beschworen, aber nicht evoziert werden.

\section{Vergangenheit und Gegenwart, Fakten und Kontrafaktisches}

Was für ein Geschichtsbild vermittelt dieses Buch vom Jahr 1913? Es wurde anfangs bereits bemerkt, dass hier, anders als in den anderen, ähnlich strukturierten Geschichtsdarstellungen, nicht eine der Katastrophen des 20. Jahrhunderts als Thema gewählt wurde, sondern eine vergleichsweise ruhige und konstruktive Phase. Interessanterweise trägt das Werk den Untertitel „Der Sommer des Jahrhunderts“ und entspricht damit dem Titelbild, das ein idyllisches Kinder-Urlaubs-Foto im strahlenden Sonnenschein zeigt. Im Augustkapitel zitiert der Autor Musils Romananfang, in dem ein schöner Augusttag des Jahres 1913 evoziert wird, wiewohl der Verfasser ausführt, der Sommer des Jahres 1913 sei der kälteste des 20. Jahrhunderts gewesen: „Es war der kälteste August des gesamten 20. Jahrhunderts. Gut, dass das die Menschen 1913 noch nicht wussten.“ (199). Da der Titel sich folglich allenfalls ironisch auf die klimatische Situation beziehen kann, aber andererseits ein durchweg emphatisches Bild von der kulturellen „Blüte“ dieses Jahres zeichnet, dürfen wir annehmen, dass er sich symbolisch auf das Jahr selbst bezieht: 1913 war der Sommer des 20. Jahrhunderts. Mit seiner künstlerischen Fruchtbarkeit wird es als die schönste und vitalste Zeit dieses von Zerstörung, Tod und „kaltem Krieg“ gekennzeichneten Jahrhunderts dargestellt. Wenn man im Bild bleibt, darf man aber auch hoffen, dass es in der behaupteten Zyklizität von Geschichte auch wieder zu milderen und reiferen Epochen kommen muss, dass also die großen Katastrophen unseren Glauben an die menschliche Zivilisation nicht für immer zerstört haben, wie die diversen kritischen Theorien des letzten Jahrhunderts von Benjamin bis Agamben konstatiert haben. 
Galle, H. -Geschichtsdarstellung in der Gegenwartsliteratur

Dass es unsere Gegenwart ist, die ein solches goldenes Zeitalter wieder heraufführt, möchte angesichts der nach der Jahrtausendwende sich überstürzenden Katastrophen und Krisen eigentlich niemand glauben. Andererseits schließt Illies` Text immer wieder diese ferne Vergangenheit mit unserer Gegenwart kurz, indem er gerade die Figuren, Ereignisse und Werke darstellt, die einen hohen Stellenwert für unsere aktuelle Kultur haben. Zugleich werden die Probleme der damaligen begrifflich auf unsere eigenen zurückgeführt („Neurasthenie“ = „burn out“). Das bewirkt, dass wir das alles kennen und auch das wiederzuerkennen glauben, was uns unbekannt war. Ja, wir mögen uns sogar in vielem überlegen fühlen, denn wir sind uns ja so vieler Dinge schon sicher, die damals noch zweifelhaft waren und erkämpft werden mussten. Die kleinen Erzählungen machen uns die Vergangenheit nicht fremd sondern vermitteln uns das Gefühl, sie sei uns nah, näher als das Grauen, das als Abgrund tatsächlich zwischen uns und dieser Vergangenheit liegt, zumal sie uns immer wieder im Präsens der aktuellen Berichterstattung vor Augen geführt wird und bruchlos in unsere frames passt.

Eingetaucht in den Horizont der Gleichzeitigkeit von 1913 kann der Leser sich sogar möglicherweise eine alternative Zukunft vorstellen, in der es nicht zu Weltkriegen, totalitären Diktaturen und Genoziden kommt, auf die ja zu jenem Zeitpunkt noch nicht viel hindeutet. In einem Interview (KLUGE 2013) äußerte Illies hierzu:

\footnotetext{
Wenn dieses Unglück von 1914 nicht stattgefunden hätte, wenn der Konjunktiv schärfer gewesen wäre, dann wäre 1914 kein Krieg ausgebrochen. Dann hätten wir kein 1933 gehabt. Wir hätten kein Auschwitz, sondern eine Welt, die den Reichtum der Zeit vor 1914 gewahrt hätte, ihren letzten Sommer zum Ausgangspunkt macht. Das wäre eine andere Wirklichkeit.
}

Das Gedankenexperiment ist eine kontrafaktische Geschichtsvorstellung, wie sie in letzter Zeit von zahlreichen Romanciers mit ganz unterschiedlichen Prämissen unternommen wurde: ein Europa, in dem Nazideutschland den Krieg gewonnen hat, bei Robert Harris‘ Fatherland, Judenverfolgung unter Präsident Charles Lindbergh in Philip Roth“ The Plot Against America, die bolschewistische Revolution in der Schweiz anstatt in Russland in Christian Krachts Ich werde hier sein im Sonnenschein und im Schatten. Alle diese Fiktionen entwerfen eine Welt, die sich erheblich von der unterscheidet, in der wir leben, aber Illies ‘ Phantasie dürfte die radikalste sein, denn die Autoren der genannten Romane lassen alle die große Katastrophe eintreten, wenn auch 
Galle, H. -Geschichtsdarstellung in der Gegenwartsliteratur

auf andere Weise oder mit anderem Ausgang. Sich vorzustellen, sie wäre nicht geschehen, scheint allzu vermessen, zu sehr als Wunschdenken ohne Bodenhaftung. Es mag sein, dass wir doch zu sehr von teleologischen Vorstellungen durchdrungen sind und deshalb den meisten eine alternative Moderne ohne Massenvernichtung als naiver Wunschtraum erscheint. Aber im Grunde ist das Buch von Illies genau dies: ein kontrafaktisches Angebot. Ganz ohne einen erfundenen Geschichtsverlauf ermöglicht es dem Leser, seine eigene Gegenwart in der jener hitzigen Belle Epoque zu spiegeln, in der die bösen Diktatoren eher als harmlose Clowns auftreten und ihre Herrschaft ein böser Traum bleibt. Die „Welt von Gestern“, jenes weitgehend friedliche, betriebsame, bürgerliche Jahrhundert Europas, das Stefan Zweig schon mit der Affäre des Oberst Redl unwiderruflich versinken sah, wird in 1913 zum Leben erweckt. Eine Welt, in der ein Schnupfen oder eine nicht mehr erwiderte Liebe schon zu den größten denkbaren Unfällen gehören, lässt uns milde lächeln, weil wir Schlimmeres gewöhnt sind, aber zugleich erfüllt sie uns mit Stolz, denn dieser ganze „Reichtum“ ist ja nicht verloren, sondern er füllt unsere Museen und Bibliotheken mit einem Glanz, an dem wir alle teilhaben können. Und außerdem glauben wir zu wissen, dass es sich nicht um eine Romanerfindung handelt, sondern um eine - wie immer amüsant gestaltete - Erzählung von Dingen, die sich wirklich zugetragen haben. Die weiträumige positive Resonanz, die das Buch gefunden hat, lässt nicht zuletzt darauf schließen, dass für diese Art von kontrafaktischer Imagination ein erheblicher Bedarf besteht.

\section{Literaturverzeichnis}

BASSLER, Moritz. Der deutsche Poproman. Die neuen Archivisten. München: Beck, 2005.

. "Zwischen den Texten der Geschichte. Vorschläge zur methodischen Beerbung des New Historicism." In: Fulda, Daniel (ed.). Literatur und Geschichte. Ein Kompendium zu ihrem Verhältnis von der Aufklärung bis zur Gegenwart. Berlin, New York: De Gruyter, 2002, 87-100.

BECKER, Sabina / KIESEL, Helmuth (ed.). Literarische Moderne. Begriff und Phänomen. Berlin: De Gruyter, 2007.

BRAUN, Michael. "Kulturgeschichte als Mosaik: Florian Illies' Jahrestagebuch erzählt aus dem Jahr 1913." Literaturkritik.de 12, 2012.

ETTE, Ottmar. "Alexander von Humboldt in Daniel Kehlmanns Welt." Humboldt im Netz XIII, no. 25, 2012, 34-40. http://www.unipotsdam.de/u/romanistik/humboldt/hin/pdf/hin25/hin_25_komplett.pdf. Acesso em: 10 de out. 2013. 
Galle, H. -Geschichtsdarstellung in der Gegenwartsliteratur

GRIMM, Florian. Reise in die Vergangenheit, Reise in die Fantasie? Tendenzen des postmodernen Geschichtsromans. Frankfurt a. M.: Peter Lang, 2008.

GUMBRECHT, Hans Ulrich. 1926. Ein Jahr am Rand der Zeit. Frankfurt a. M.: Suhrkamp, 2003.

HoLl, Frank. "„Die zweitgrößte Beleidigung des Menschen sei die Sklaverei ...“. Daniel Kehlmanns neu erfundener Alexander von Humboldt." Humboldt im Netz XIII, no. 25, 2012, 46-62.

Hutcheon, Linda. A Poetics of Postmodernism. History, Theory, Fiction. London, New York: Routledge, 1991.

ILLIES, Florian. 1913. Der Sommer des Jahrhunderts. Frankfurt a. M.: Fischer, 2012.

KEUSCHNIG, Gregor. "Florian Illies: 1913." In: Begleitschreiben (2013). Published 02.01.2013. http://www.begleitschreiben.net/florian-illies-1913/. Acesso em: 12 de out. 2013.

KLUGE, Alexander. "Herr Illies, wie war das Jahr 1913?" In: Welt am Sonntag, 12.01.2013.

KNOBLOCH, Eberhard. "Alexander von Humboldt und Carl Friedrich Gauß." Humboldt im Netz XIII, no. 25, 2012, 63-79.

MARX, Friedhelm. "Die Vermessung der Welt als historischer Roman." In: NICKEL, Gunther (ed.). Daniel Kehlmanns Die Vermessung Der Welt. Materialien, Dokumente, Interpretationen. Reinbek bei Hamburg: Rowohlt, 2009, 169-85.

MoRTON, Frederic. Thunder at Twilight: Vienna 1913/14. Cambridge: Da Capo Press, 2001.

OORT, Frans. "Measuring the World." Notices of the AMS 55, no. 6, 2008, 681-84.

PEREIRA, Valéria Sabrina. "Orquestrando ecos do passado. Walter Kempowski e Das Echolot." Dissertation, USP, 2011.

PfoHLMANN, Oliver. "Eine nervöse, überdrehte Zeit." In: Neue Zürcher Zeitung, 05.12.2012.

SCHILLING, Erik. Der Historische Roman seit der Postmoderne. Umberto Eco und die deutsche Literatur. Heidelberg: Winter, 2012.

SEIBT, Gustav. "Am Vorabend der Katastrophe." In: Süddeutsche Zeitung, 25.10.2012.

TROTHA, Hans von. "Funkelnde Tage. Florian Illies erklärt uns das erstaunliche Jahr 1913." In: Die Zeit, 10.12.2012.

WALLASCH, Alexander. "Man umarmt sich herzlich. Florian Illies tickert in „1913. Der Sommer des Jahrhunderts" kurze Episoden aus dem Leben von Benn, Rilke, Kafka und anderen." In: taz, 25.10.2012.

Walton, Kendall. Mimesis as Make-Believe. On the Foundations of the Representational Arts. Cambridge, Massachussetts: Harvard University Press, 1990.

WeBER, Thomas. "Hinterher ist vergessen, dass man vorher nichts wusste." In: Frankfurter Allgemeine Zeitung, 01.12.2012, L 14.

recebido em 10 de março de 2014

aceito em 03 de maio de 2014

Pandaemonium, São Paulo, v. 17, n. 23, Jun./2014, p. 26-49 